\title{
Paracardial fat and vitamin A: a mechanism for regulating exercise performance
}

\author{
Leroy C. Joseph and John P. Morrow \\ Division of Cardiology and Department of Medicine, Columbia University College of Physicians and Surgeons, New York, New York, USA.
}

\begin{abstract}
Different fat depots have different physiologic functions. In a provocative study published in this issue of the $J C l$, Petrosino et al. investigate the role of paracardial fat in whole-body metabolism and exercise physiology. Petrosino et al. show that paracardial fat samples from older mice or mice fed a Western diet had decreased levels of alcohol dehydrogenase 1 (ADH1). Paracardial fat samples from humans with obesity also had decreased levels of $A D H 1$ mRNA, supporting the translational relevance. Additional experiments with Adh1-KO mice and surgical fat transplantation experiments provide additional mechanistic insight. Paracardial fat may regulate exercise performance by altering circulating metabolites and/or endocrine effects. ADH1 appears to regulate the mitochondrial content of paracardial fat, a mechanism mediated by retinaldehyde. When ADH1 is active, the paracardial fat has characteristics of brown fat, which is beneficial for exercise performance. Further research is warranted to determine the translational potential of these findings, such as whether removing paracardial fat at the time of open-heart surgery might improve recovery time by increasing exercise capacity.
\end{abstract}

\section{Paracardial fat mass}

There is an extensive body of literature showing that different fat depots have different physiologic functions (1). Far from being a passive storage space for excess calories, adipose tissue can influence circulating hormonal and inflammatory pathways (2). Years of clinical research have focused on epicardial fat, which correlates with an increased risk of cardiovascular events $(3,4)$. In a provocative study published in this issue of the JCI, Petrosino et al. investigate the role of paracardial fat in whole-body metabolism and exercise physiology (5). Paracardial fat is relatively under-studied compared with other fat depots. A moment regarding terminology: although usage varies, most authors define epicardial fat as fat within the pericardium and paracardial fat as superficial to the pericardium, often around the atria and great vessels. The term pericardial fat is sometimes used to refer to both epicardial and paracardial fat (6). Mice, in general, lack visible amounts of epicardial fat, but they can have abundant paracardial fat depending on age, diet, and genotype. This heart-associated fat can actually be quite a nuisance for those of us who frequently dissect out the heart and aorta for experiments.

Petrosino et al. found that paracardial fat mass increased with aging and also after high-fat diet feeding. Further, the histology of the adipocytes shifted to a more white fat morphology with aging and a high-fat diet (specifically, the authors found larger, unilocular adipocytes under these conditions) compared with adipocytes in control paracardial fat (5).

\section{Exercise performance}

In this study, a larger paracardial fat mass was associated with decreased exercise performance (Figure 1). Exercise physiology is a complex phenomenon that integrates pulmonary, cardiac, and skeletal muscle function as well as autonomic nervous system inputs and release of metabolites from the liver as a fuel source. Impairment of any of these physiologic functions could reduce exercise performance. Although much of the general population is fascinated by exercise performance in the context of college sports, professional sports, the Olympics, etc., few adults in Western societies are themselves compliant with evidence-based recommendations for regular exercise (7). There is some evidence from clinical studies that exercise can reduce epicardial fat (8), but could the reverse be true as well - that is, could paracardial fat reduce exercise performance?

Petrosino et al. used RNA-Seq to examine gene expression in paracardial fat compared with intra-abdominal fat. The authors reported increased mRNA levels of genes involved in vitamin A (retinol) metabolism, focusing in particular on alcohol dehydrogenase 1 (ADH1) (5). Vitamin A is thought to have an important role in obesity and diabetes, although much of the literature is contradictory as to the details (9). ADH1 causes the oxidation of retinol into retinaldehyde (aka, retinal), which is then converted to retinoic acid. Retinoic acid has diverse biologic effects that are largely mediated by the retinoic acid receptor (RAR) family of transcription factors. Petrosino et al. showed that paracardial fat samples from older mice or mice fed a Western diet had decreased levels of $\mathrm{ADH} 1$ and that paracardial fat samples from humans with obesity also had decreased levels of ADH1 mRNA (5).

The mechanism for paracardial fat remodeling

Additional experiments with Adh1-KO mice provided more mechanistic insight. These Adh1-KO mice had larger paracardi- 


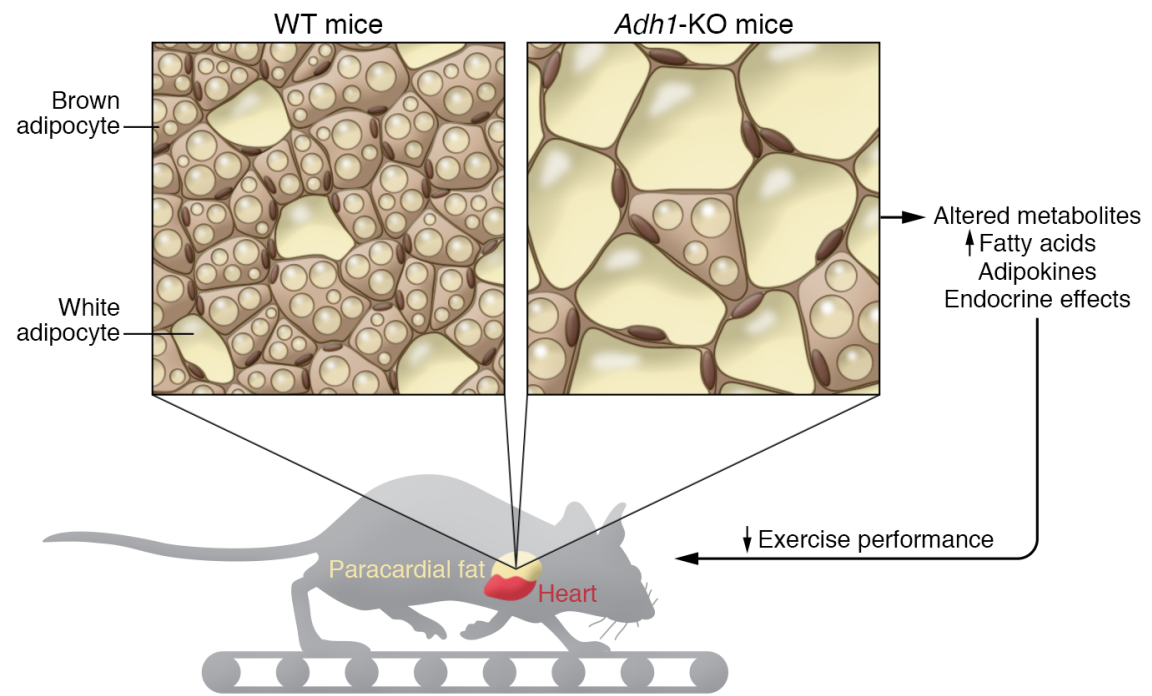

al fat mass without changes in heart mass or body weight. Food intake and fasting glucose levels were similar between Adh1KO and WT mice. However, the Adh1-KO mice had decreased exercise performance. Serum metabolites indicated increased circulating fatty acid levels in the Adh1KO mice compared with control. Thus, increased paracardial fat mass was correlated with decreased exercise performance under several conditions. A series of surgical experiments demonstrated that this decrease was not just a correlation. Surgical removal of paracardial fat in Adh1KO normalized serum metabolites and increased maximum oxygen consumption during exercise. The authors took the additional step of performing a series of paracardial fat transplantation experiments, surgically transplanting either WT paracardial fat or Adh1-KO paracardial fat into WT mice. The presence of paracardial fat from Adh1-KO mice caused a marked reduction in maximum oxygen consumption during exercise compared with transplantation of WT paracardial fat (5). This is strong evidence, rather than a correlation, that paracardial fat tissue played a causal role in the decrease in exercise performance.

In the last part of the article, the authors propose that retinol metabolism regulates paracardial fat remodeling through mitochondrial mechanisms. Electron microscopy showed that paracardial adipocytes from Adh1-KO mice had smaller mitochondria, and at the protein level, there was reduced expression of optic atrophy 1 (Opa1), which regulates mitochon- drial fusion. Similar findings were shown in paracardial samples from humans, in that samples from humans with obesity had decreased mitochondrial content and lower OPA1 expression levels. In vivo retinaldehyde treatment in mice recruited PGC- $1 \alpha$ to adipocyte nuclei, which should lead to the increased transcription of genes associated with mitochondrial function and biogenesis. Opa1 mRNA was in fact increased by in vivo retinaldehyde treatment, with increased mitochondrial DNA content in paracardial fat from WT mice. This may represent a browning of the paracardial fat. However, these changes were independent of uncoupling protein 1 (UCP1) expression, the classic marker for brown fat, so the paracardial fat may have been a form of beige fat (5).

\section{Conclusions}

Like all innovative science, this study raises at least as many questions as it answers. One of the questions raised is: How does the retinaldehyde/ADH1 pathway regulate PGC- $1 \alpha$ ? PGC- $1 \alpha$ can act as a coactivator for several transcription factors, including PPARs. PPARs have important regulatory roles for many metabolic genes. PPAR $\gamma$, in particular, is known to have an important role in adipocyte biology (10). Therefore, PPARs probably play an important role in the regulation of paracardial fat. PPARs heterodimerize with retinol $\mathrm{X}$ receptors (RXRs) to release corepressors and bind PCG1a. Subsequent activation of downstream genes leads to differential regulation by the PPAR isoforms interacting with
Figure 1. ADH1 impacts paracardial fat mass. Petrosino et al. (5) showed that Adh1-KO mice had larger paracardial fat mass with changes in adipocyte histology, without changes in heart mass or body weight. Food intake and fasting glucose levels were similar to in both Adh1-KO and WT mice. However, the Adh1-KO mice had a decrease in exercise performance. Serum metabolites indicated increased circulating fatty acid levels in the Adh1-KO mice compared with controls.

RXRs. This pathway could be an interesting direction for future work.

Another important question: Does paracardial fat influence exercise performance by acting as an endocrine organ, or by releasing metabolites that affect cardiac and/or skeletal muscle? These explanations are not mutually exclusive. It should also be noted that obesity and a high-fat diet cause excessive lipid deposition inside myocytes, which can contribute to pathophysiology (11), and chronic elevation of serum fatty acids in the Adh1-KO mice might mimic these effects, but this was not the focus of the work under discussion. Interestingly, there is prior work showing that brown adipose tissue can decrease exercise performance by releasing myostatin (12), so different fat depots may have opposite effects on muscle function. These opposing effects highlight the current findings (5) that paracardial fat could have unique properties.

A limitation of this study is the use of a full-body Adh1-KO model. Although the authors demonstrate that the liver and skeletal muscle appear normal by histology (5), some biochemical role for ADH1 in these tissues, or the heart itself, may exist. Adult myocardium is known to respond to retinoic acid (13). Furthermore, decreased retinoid receptor signaling is believed contribute to diabetic cardiomyopathy (14). Future work with tissue-specific KO animals might refine the working model and give us more information about the relative contribution of skeletal muscle and cardiac function with regard to exercise performance. 
Petrosino et al. (5) should be congratulated on their innovative contribution to our understanding of how paracardial fat can influence exercise performance and on the proposed role of vitamin A as the mechanism of paracardial fat remodeling. This vitamin A mechanism is an important advance in our understanding of the interplay between fat depots, circulating metabolites, and exercise performance. More research is needed to better understand the molecular mechanisms and determine the translational potential of these findings. For example, we might hypothesize that removal of paracardial fat at the time of open-heart surgery could improve recovery time by increasing exercise capacity.

\section{Acknowledgments}

JPM is supported by NIH grant R01 HL136758.
Address correspondence to: John P. Morrow, PH10-203, College of Physicians and Surgeons of Columbia University, $650 \mathrm{~W}$ 168th Street, New York, New York 10032, USA. Email: jpm46@cumc. columbia.edu.

1. Cohen P, Spiegelman BM. Cell biology of fat storage. Mol Biol Cell. 2016;27(16):2523-2527.

2. Weisberg SP, et al. Obesity is associated with macrophage accumulation in adipose tissue. J Clin Invest. 2003;112(12):1796-1808.

3. Iacobellis G. Epicardial fat: a new cardiovascular therapeutic target. Curr Opin Pharmacol. 2016;27:13-18.

4. Al Chekakie MO, et al. Pericardial fat is independently associated with human atrial fibrillation. J Am Coll Cardiol. 2010;56(10):784-788.

5. Petrosino JM, et al. Paracardial fat remodeling affects systemic metabolism through alcohol dehydrogenase 1. JClin Invest. 2021;131(4):e141799.

6. Iacobellis G. Epicardial and pericardial fat: close, but very different. Obesity (Silver Spring). 2009;17(4):625-627.

7. Piercy KL, Troiano RP. Physical activity guide- lines for Americans from the US Department of Health and Human Services. Circ Cardiovasc Qual Outcomes. 2018;11(11):005263.

8. Saco-Ledo G, et al. Physical exercise and epicardial adipose tissue: a systematic review and meta-analysis of randomized controlled trials. Obes Rev. 2020;22(1):13103.

9. Blaner WS. Vitamin A signaling and homeostasis in obesity, diabetes, and metabolic disorders. Pharmacol Ther. 2019;197:153-178.

10. Rosen ED, et al. PPAR gamma is required for the differentiation of adipose tissue in vivo and in vitro. Mol Cell. 1999;4(4):611-617.

11. Gowen BH, et al. Mechanisms of chronic metabolic stress in arrhythmias. Antioxidants (Basel). 2020;9(10):E1012.

12. Kong X, et al. Brown adipose tissue controls skeletal muscle function via the secretion of myostatin. Cell Metab. 2018;28(4):631-643.

13. Asson-Batres MA, et al. Effects of vitamin A deficiency in the postnatal mouse heart: role of hepatic retinoid stores. Am J Physiol Heart Circ Physiol. 2016;310(11):H1773-H1789.

14. Pan J, et al. Molecular mechanisms of retinoid receptors in diabetes-induced cardiac remodeling. J Clin Med. 2014;3(2):566-594. 